\title{
Visual Perception in Nuclear Medicine and Volumetric Data
}

\author{
M.W. PIETRZYK* \\ The Institute of Physics, 76 Portland Place, London W1B 1NT, UK
}

\begin{abstract}
Any diagnostic decision that involves imaging techniques depends not entirely on the images themselves but also on the perception and interpretation by a radiologist. In fact, such human factors appear to be a bottleneck to imaging accuracy. A better understanding of visual perception and decision-making processes seem to be necessary for the advancement and optimization of medical imaging. Therefore, research on the accuracy of medical imaging draws on techniques from a wide range of disciplines in an attempt to minimize diagnostic errors and improve the healthcare system. This short review of the psychophysical studies in the radiological domain focuses on the recent work conducted in the visual perception of nuclear medicine images and volumetric data.
\end{abstract}

DOI: 10.12693 /APhysPolA.127.1523

PACS: 87.63.lj

\section{Introduction}

The purpose of medical imaging is to provide visual information that reduces uncertainty about the patient's state of health through perceptual inspection of such data, for which quality is restricted by image-dependent factors such as artefacts of the image-forming process, overlapping anatomy, and quantum limits [1]. Since it is presented to an observer, who does not necessary measure physical quantities like contrast, resolution, or signal-tonoise ratios, but rather sees objects and understands the meaning of their presence, the image-independent factors contribute to the imaging accuracy. The medical imaging modality can be improved by enhancement of the ability to perceive relevant, critical features in the image that could be optimized and tuned to specific requirements. The medical image perception has been developing since the mid-20 century [2], and a milestone in theoretical framework was achieved with an application of receiver operating characteristics (ROC) that adapted signal detection theory for quantification of diagnostic performance $[3,4]$.

\section{ROC}

A recent example of the $\mathrm{ROC}$ multimodality study was about the impact of hybrid single photon emission computed tomography/computed tomography (SPECT/CT) on the detection of a parathyroid adenoma [5]. Parathyroid disease affects approximately one in 500 women and one in 2,000 men in their fifth to seventh decades, with parathyroid adenoma accounting for $80 \%-85 \%$ of those cases [6]. The SPECT/CT is highly appreciated for correctly localizing gonads [7], which is especially useful for surgery and offers some define advantages over planar scintigraphy [8]. As a limited benefit was reported of

\footnotetext{
*e-mail: dr.mariusz.pietrzyk@gmail.com mariusz.pietrzyk@physics . org
}

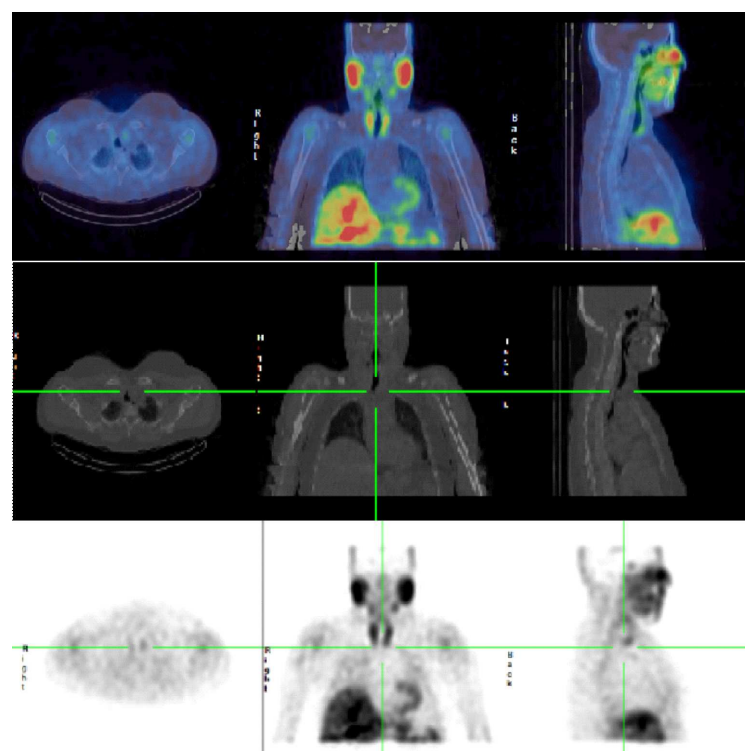

Fig. 1. Examples of images used by Morrison et al. [5], i.e., hybrid SPECT/CT (at the top), CT only (in the middle), and SPECT only (at the bottom).

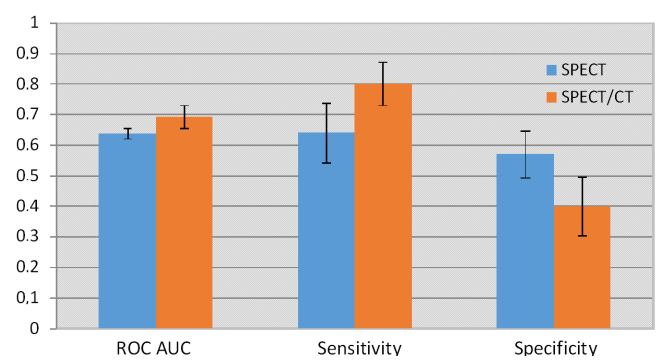

Fig. 2. Mean performance metrics and standard deviation (error bars) of ROC AUC, sensitivity, and specificity.

additional CT information for the detection of parathyroid lesions within the neck using the hybrid modality [9-12], Morrison et al. [5] conducted a quantitative study on how additional CT information when added to SPECT effects reader performance based on six negative and 10 suspected single parathyroid adenoma cases. 
In this study, five nuclear medicine specialists analyzed the same 16 cases, ordered randomly, under two conditions. First, SPECT scans with associate planar images were presented (Fig. 1). Second, SPECT and CT scans were available with associate planar images, and the image fusion tool (Fig. 1). ROC methodology requires a confidence ranking associated with each decision, and radiologists used a 10-level scale to evaluate each case within an unlimited time. The CT information implies a trend of increased confidence in decision-making, which had miscellaneous effects on performance evaluation. The radiologists improved detection sensitivity, but also increased the recall error rate (Fig. 2), which has not been previously reported in the literature. The implications of the study were appreciated by clinicians and perceptual communities.

\section{Eye-tracking}

Humans are inherently good at interpreting visual information, and the ROC methodology quantified the differences between the observers' performances based on final outcomes made among a series of cases. However, the investigation of the decision-making process requires additional scientific methods. Recoding the eye movements of observers when they look at visual scenes gives insight into what is attracting their attention [13]. Eyetracking experiments enable the study of visual searching strategies [14] and foster investigation on human limitations in the detection of pathology [15]. Early studies showed that human error was related to perceptual and cognitive aspects [16], and the correct decision is generally made within the first seconds of viewing images [17].

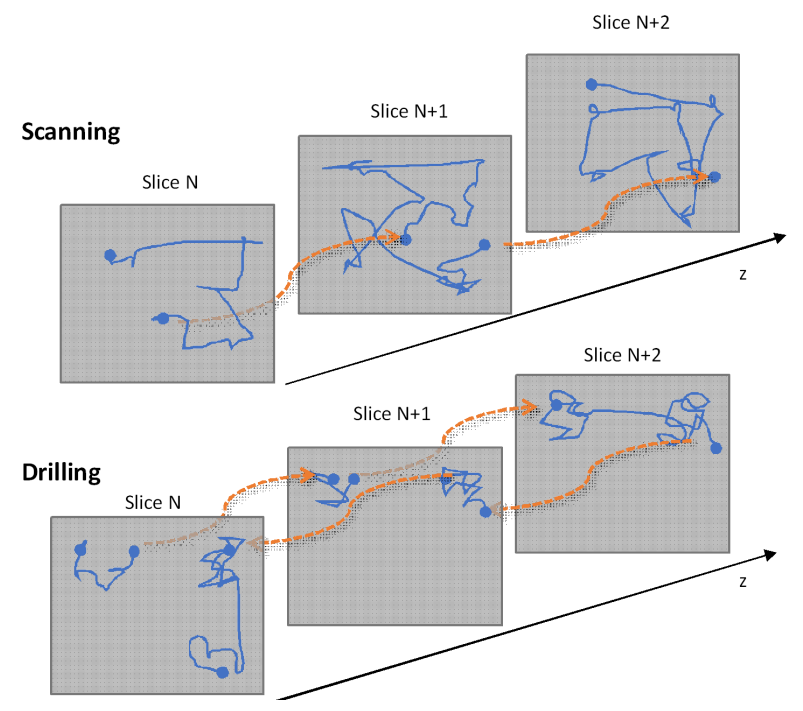

Fig. 3. Typical visual searching strategies applied for volumetric data, i.e., scanning each slice (at the top) and drilling back and forward between slices (at the bottom). Blue lines represent eye-tracking recordings, and the orange arrows between slices indicate link position on previous and next slices.
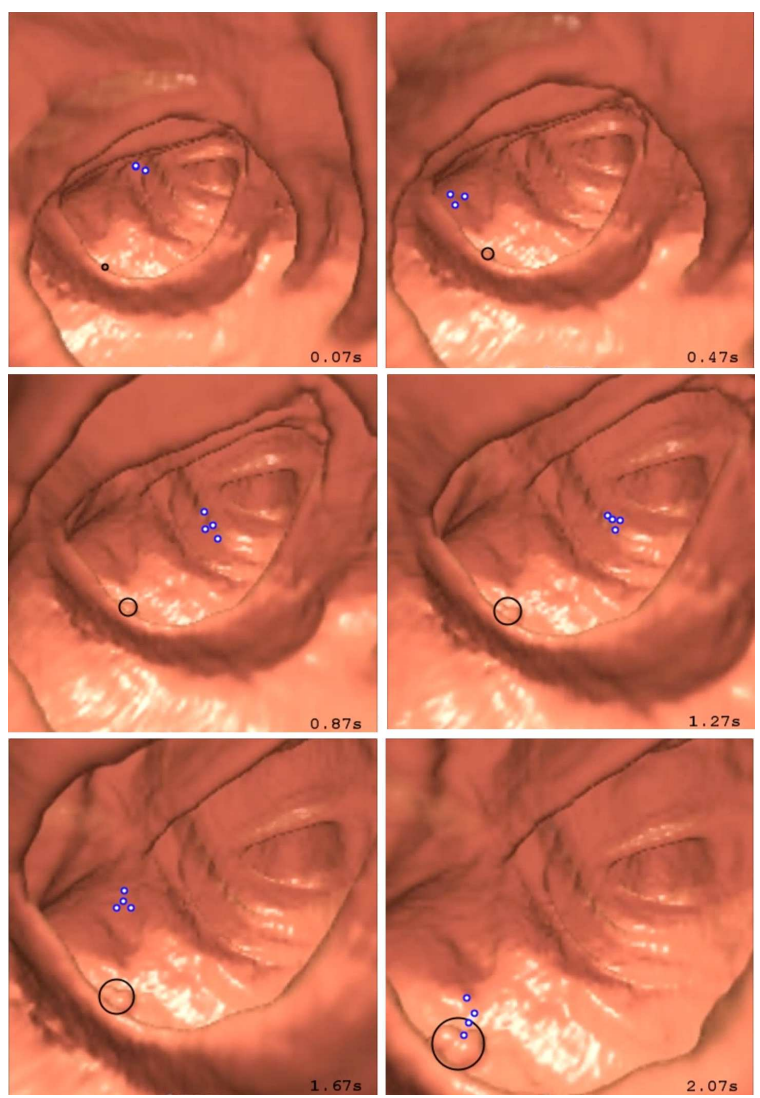

Fig. 4. Print screen of a fly-through CT colonoscopy the frame-by-frame visualizations shows a colon polyp (within black circle) and the current location of eye fixations (series of blue/white dots). The size of the visible part of the polyp depends on the relative distance to the target and the direction of view. The observer had focused on the polyp within $2 \mathrm{~s}$ after it appeared for the first time $(t=0.07 \mathrm{~s})$. Adapted from [25].

Pietrzyk et al. [18] showed that an initial direction of specialists' attention is correlated with pathology location. Such correlation was less typical for inexperienced observers.

Feedback of eye-tracking data to human observers facilitated the performance of radiological tasks. Perceptual enhancement of tumor targets, by highlighting the most dwelled region of interest, had improved the radiologists' overall accuracy by $16 \%$ [19]. Experts' eyetracking recordings successfully guided radiography students during interpretation of chest X-rays [20]. An error prediction algorithm based on eye-tracking data and image features analysis improved radiologists' decisionmaking process by $25 \%$ [21]. This novel computer aided detection (CAD) concept was an inspiration for further investigation [22] and might provide a solution to current CAD system, which can increase radiology sensitivity $10 \%$, but with a comparable deterioration in specificity [23]. Pietrzyk et al. (2012) proposed a method to tackle the problem of specificity reduction with CAD system [21].

Inspecting volumetric data is not a trivial task, especially when searching for a subtle sign of cancer in 

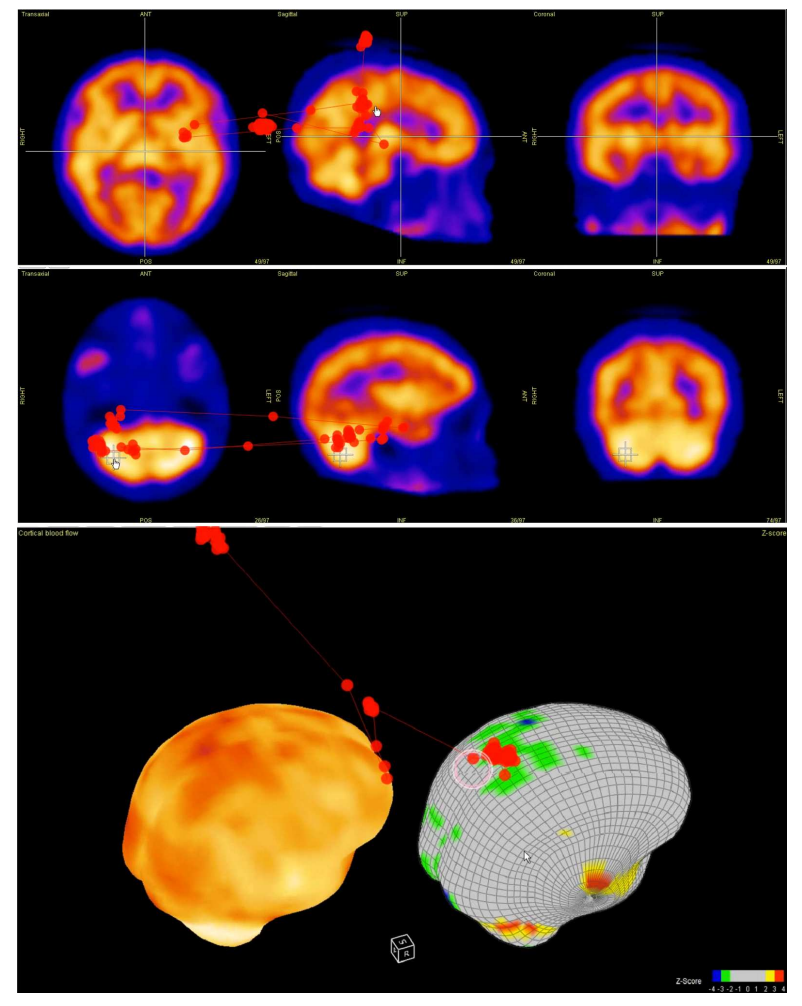

Fig. 5. Example of eye-tracking recordings (red dots) acquired during central blood flow (CBF) PET-based imaging [27, 28].

noise CT scans. In order to evaluate radiologists' ability to cope with such a challenge, Drew et al. (2013) invited 24 radiologists to search for lung nodules through a stack of CT axial slides, while monitoring eye position on the display screen. The two dominant search strategies (Fig. 3) were described. The first searching strategy, the drilling, depended on restricted searching areas on axial slides and frequent back and forward motion across the slides (in $z$-directions). In contrast, the second approach, scanning, was characterized with a constant and slow motion in a $z$-direction while analyzing the entire axial slides before moving on to the next slide. Drilling was superior to the scanning regarding the lung nodule detection rate, percentage of the lung covered, and the percentage of search errors where a nodule was never fixated [24].

Another recent eye-tracking study [25] that involved the inspection of volumetric data was based on flythrough modes of virtual colonoscopy examinations, where the abnormality (i.e., polyps) was moving and changing in size (Fig. 4). Radiologists inspected virtual colons, which were rendered from CT volumes. Phillips et al. [25] showed that experts tended to have shorter dwell times per fixations and shorter cascades amplitudes than inexperience observers. As this contradicted previous studies on 2D modalities [26], objectives for further medical image perception research on $3 \mathrm{D}$ data arose to in- vestigate additional perceptual-cognitive factor involved in the inspection of multidimensional and multimodality data (Fig. 5). As such, the restriction in spatial orientation and navigation skills appears to be an additional source of human limitations.

\section{Conclusion}

New medical imaging modality, or its novel part, needs to be evaluated before it can be accepted for a clinical application. Medical image perception is an interdisciplinary domain that targets such a problem with the appropriate methodology. In particular, the accuracy of the overall diagnostic performance is quantified with ROC, and the decision-making process is monitored accordingly to visual attention distribution and cognitive overload. In addition, perceptual studies contributed to a better understanding of human factors involved in radiological tasks and provide a foundation for error management.

\section{References}

[1] D.J. Manning, A. Gale, E.A. Krupinski, Brit. J. Radiol. 78(932), 683 (2005).

[2] C.C. Birkelo, W. Chamberlain, P. Phleps, P. Schools, D. Zacks, J. Yerushalmy, J. Am. Med. Assoc. 133, 359 (1947).

[3] C.E. Metz, Semin. Nucl. Med. 8, 283 (1978).

[4] D.M. Green, J.A. Swet, Signal Detection Theory and Psychophysics, Krieger, Huntington NY 1974.

[5] A. Morrison, P. Brennan, W. Reed, M. Pietrzyk, G. Schembri, E. Bailey, P. Roach, M. Evanoff, P. Kench, SPIE Med. Imag. 7966 , 79661J (2011).

[6] A.G. Kettle, M.J. O'Doherty, Semin. Nucl. Med. 36, 206 (2006).

[7] D. Papathanassiou, J. Flament, J. Pochart, M. Patey, H. Marty, J. Liehn, C. Schvartz, Clin. Nucl. Med. 33, 394 (2008).

[8] M. Lorberboym, I. Minski, S. Macadziob, G. Nikolov, P. Schachter, J. Nucl. Med. 44, 904 (2003).

[9] W.C. Lavely, S. Goetze, K. Friedman, J. Leal, Z. Zhang, E. Garret-Mayer, A.P. Dackiw, R.P. Tufano, M.A. Zeiger, H.A. Ziessman, J. Nucl. Med. 48, 1084 (2007).

[10] I.W. Gayed, E. Kim, W. Broussard, D. Evans, J. Lee, L. Broemeling, B.B. Ochoa, D.M. Moxley, W.D. Erwin, D.A. Podoloff, J. Nucl. Med. 42, 76 (2005).

[11] Y. Krausz, L. Bettman, L. Guralnik, G. Yosilevsky, Z. Keidar, R. Bar-Shalom, E. Even-Sapir, R. Chisin, O. Israel., World J. Surg. 30, 76 (2006).

[12] A. Serra, P. Bolasco, L. Satta, A. Nicolosi,8 A. Uccheddu, M. Piga, Radiolog. Med. 111, 999 (2006).

[13] M. Pietrzyk, D.J. Manning, T. Donovan, A. Dix, SPIE Med. Imag. $\mathbf{7 6 2 7}, 726719$ (2010).

[14] E.A. Krupinski, Acad. Radiol. 3, 137 (1996).

[15] M. Pietrzyk, T. Donovan, P. Brennan, A. Dix, D.J. Manning, SPIE Med. Imag. 7966, 79660A (2011). 
[16] H.L. Kundel, C.F. Nodine, D. Carmody, Investigate Radiol. 13, 175 (1978).

[17] C.F. Nodine, C. Mello-Thoms, H.L. Kundel, S.P. Weinstein, Am. J. Roentgenol. 179, 917 (2002).

[18] M. Pietrzyk, M. McEntee, M. Evanoff, P. Brennan, C. Mello-Thoms, SPIE Med. Imag. 9037, 90371A (2014).

[19] E.A. Krupinski, C.F. Nodine, H.L. Kundel, Percept. Psychophys. 53, 519 (1993).

[20] D. Litchfield, L.J. Ball, T. Donovan, D.J. Manning, T. Crawford, SPIE Med. Imag. 6917,691715 (2008).

[21] M. Pietrzyk, D. Rannou, P. Brennan, SPIE Med. Imag. 8318, 831815 (2012).

[22] S. Voisin, F. Pinto, G. Morin-Ducote, B. Kathleen, B. Hudson, G.D. Tourassi, Med. Phys. 40, 101906 (2013).
[23] R.M. Nishikawa, R. Schmidt, M. Linver, A. Edwards, J. Papaioannou, M. Stull, Am. J. Roentgenol. 198, 708 (2012).

[24] T. Drew, M. Vo, A. Olwal, F. Jacobson, S. Seltzer, J. Wolfe, J. Vision 13, 1 (2013).

[25] P. Phillips, D. Boone, S. Mallett, S. Taylor, D. Altman, D. Manning, A. Gale, S. Halligan, Radiology 267, 924 (2013).

[26] M. Pietrzyk, D.J. Donovan, T. Manning, A. Dix, SPIE Med. Imag. 7263, 72630I (2009).

[27] J.J. Chen, M. Wieckowska, E. Meyer, G.B. Pike, Int J. Biomed. Imag. 2008, 516359 (2008).

[28] G. Kägi, K.P. Bhatia, E. Tolosa, J. Neurol. Neurosurg. Psychiatry 81, 5 (2010). 